\title{
EPI-DERIVATIVES OF INTEGRAL FUNCTIONALS WITH APPLICATIONS
}

\author{
PHILIP D. LOEWEN AND HARRY H. ZHENG
}

\begin{abstract}
We study first- and second-order epi-differentiability for integral functionals defined on $L^{2}[0, T]$, and apply the results to obtain first- and second-order necessary conditions for optimality in free endpoint control problems.
\end{abstract}

\section{INTRODUCTION}

The notion of epi-differentiability for nonsmooth functions with extended real values was introduced and developed by Rockafellar $[12,13]$. He shows $\left[12\right.$, Theorem 4.5] that a sufficient condition for $f: \mathbb{R}^{m} \rightarrow \mathbb{R} \cup\{+\infty\}$ to be twice epi-differentiable at the point $x$ is the existence of a local representation $f=g \circ F$ in which $F: \mathbb{R}^{m} \rightarrow \mathbb{R}^{d}$ is a $C^{2}$ mapping and $g: \mathbb{R}^{d} \rightarrow \mathbb{R} \cup\{+\infty\}$ is a piecewise linear-quadratic convex function such that $g(F(x))$ is finite and a certain constraint qualification holds. Under these conditions $f$ is called fully amenable at $x$. A large class of finite-dimensional optimization problems can be described in terms of fully amenable functions. Poliquin and Rockafellar $[9,10]$ develop a calculus of epi-derivatives and apply it to derive optimality conditions for certain mathematical programming problems.

Epi-differentiability has also been studied in reflexive Banach spaces. Do [5] treats convex integral functionals, while Cominetti [4] considers general amenable functions. A discussion of their results, of Noll's recent work [8], and of Levy's concurrent research on the same topic [6], appears at the end of Section 3.

In this paper, we study the epi-differentiability of the extended-valued functional $\mathscr{F}$ defined on $L^{2}\left([0, T] ; \mathbb{R}^{m}\right)$ by

$$
\mathscr{I}(u)=\int_{0}^{T} f(t, u(t)) d t
$$

where the integrand $f(t, \cdot)$ is, for almost all $t$, a fully amenable function of a particular form, namely, the sum of a finite-valued fully amenable function and an indicator function. (See Section 3 for details.) We show that the the

Received by the editors January 5, 1993.

1991 Mathematics Subject Classification. Primary 49J52, 49K05; Secondary 58C20, 58C05.

Key words and phrases. Integral functionals, epiderivative, epiconvergence.

The first author's research was supported by the Natural Science and Engineering Research Council (NSERC) of Canada through grant 5-89441. 
first and second epi-derivatives of $\mathscr{I}$ can be expressed in terms of the epiderivatives of $f(t, \cdot)$-effectively justifying the operation of "differentiating under the integral sign". This reduces our infinite-dimensional differentiation problem to a finite-dimensional one for which results are already available, and lets us obtain optimality conditions for minimization problems involving $\mathscr{I}$. We also discuss the epi-differentiability of simple composite functionals involving $\mathscr{I}$, particularly Bolza functionals. We then apply our results to derive second-order necessary conditions for free endpoint control problems. Since epi-differentiability has a strong geometric foundation and can capture the local behavior of integral functionals near a given point, this method may also be useful in studying optimality conditions for problems with constraints on both endpoints.

We begin with some background material, then study the epi-differentiability of the nonconvex integral functional $\mathscr{I}$, and finally apply our results to a Bolza functional.

\section{BACKGROUND MATERIAL}

We write $\overline{\mathbb{R}}$ for the extended real line $\mathbb{R} \cup\{+\infty\}$. Throughout this section, $(X,\|\cdot\|)$ is a normed linear space; a function $f: X \rightarrow \overline{\mathbb{R}}$ is called proper when its domain, the set $\operatorname{dom} f=\{x \in X: f(x)<+\infty\}$, is nonempty. In cases where $X$ is finite-dimensional, we think of its elements as column vectors and the elements of $X^{*}$ (e.g., gradients and normal vectors) as row vectors.

Given an extended-real-valued function $f$ on $X$ and a point $x$ in the domain of $f$, the first difference quotient for $f$ at $x$ is the function $f_{x, h}: X \rightarrow \overline{\mathbb{R}}$ defined by

$$
f_{x, h}(y)=\frac{f(x+h y)-f(x)}{h} .
$$

The epi-differentiability of $f$ at $x$ is characterized by the equation

$$
\underset{h \downarrow 0}{\limsup \operatorname{epi}} f_{x, h}=\liminf _{h \downarrow 0} \operatorname{epi} f_{x, h}=\operatorname{epi} f_{x}^{\prime} \text {. }
$$

(Recall that for any $g: X \rightarrow \overline{\mathbb{R}}$, epi $g=\{(x, r): x \in \operatorname{dom} g, r \geq g(x)\}$.) We say that $f$ is epi-differentiable at $x$ if the first equation holds and the function $f_{x}^{\prime}$ defined by the second equation obeys $f_{x}^{\prime}(0)>-\infty$. In this case the function $f_{x}^{\prime}$ is lower semicontinuous and satisfies $f_{x}^{\prime}(0)=0$ : it is called the epi-derivative of $f$ at $x$. This is the Kuratowski-Painleve mode of epi-convergence, in which the strong topology is used throughout. Thus criteria (I) and (II) below are both equivalent to (1).

(I) For any $y \in X$ and any sequence $h_{i} \downarrow 0$ we have both

(i) Any sequence $y_{i} \rightarrow y$ obeys $\liminf _{i \rightarrow \infty} f_{x}, h_{i}\left(y_{i}\right) \geq f_{x}^{\prime}(y)$; and

(ii) There exists a sequence $y_{i} \rightarrow y$ such that $\lim \sup _{i \rightarrow \infty} f_{x}, h_{i}\left(y_{i}\right) \leq$ $f_{x}^{\prime}(y)$.

(II) For all $y \in X$, we have

$$
\liminf \inf _{h \downarrow 0} f_{x, h}\left(y^{\prime}\right)=\lim \sup _{h \downarrow 0} \inf _{y^{\prime} \rightarrow y} f_{x, h}\left(y^{\prime}\right)=f_{x}^{\prime}(y)
$$


where, by definition,

$$
\begin{aligned}
\liminf \inf _{h \downarrow 0} f_{y^{\prime} \rightarrow y}, h\left(y^{\prime}\right) & =\lim _{\epsilon \downarrow 0} \liminf _{h \downarrow 0} \inf _{\left\|y^{\prime}-y\right\|<\epsilon} f_{x, h}\left(y^{\prime}\right), \\
\lim \sup _{h \downarrow 0} \inf _{y^{\prime} \rightarrow y} f_{x, h}\left(y^{\prime}\right) & =\lim _{\epsilon \downarrow 0} \limsup _{h \downarrow 0} \inf _{\left\|y^{\prime}-y\right\|<\epsilon} f_{x, h}\left(y^{\prime}\right) .
\end{aligned}
$$

To arrive at a third characterization of epi-differentiability, we recall that any proper function $F: X \rightarrow \overline{\mathbb{R}}$ gives rise to a family $\left\{F^{\lambda}\right\}_{\lambda>0}$ of Moreau-Yosida approximates, where

$$
F^{\lambda}(x)=\inf _{u \in X}\left\{F(u)+\lambda\|u-x\|^{2}\right\} \quad \forall x \in X .
$$

Note that $F^{\lambda}(x)$ is nondecreasing as a function of $\lambda$. The following result of Attouch [1, Thm. 2.65] relates compound limits of the sort in criterion (II) to iterated limits involving the Moreau-Yosida approximates.

Theorem 1. Let each $F_{i}: X \rightarrow \overline{\mathbb{R}}$ be proper. If there exist $r \geq 0, x_{0} \in X$, such that

$$
\inf _{i} F_{i}(x) \geq-r\left(\left\|x-x_{0}\right\|^{2}+1\right) \quad \forall x \in X,
$$

then the following equations hold for every $x$ in $X$ :

$$
\begin{aligned}
& \lim \inf _{i \rightarrow \infty} \inf _{x^{\prime} \rightarrow x} F_{i}\left(x^{\prime}\right)=\lim _{\lambda \rightarrow \infty} \liminf _{i \rightarrow \infty} F_{i}^{\lambda}(x), \\
& \lim \sup _{i \rightarrow \infty} \inf _{x^{\prime} \rightarrow x} F_{i}\left(x^{\prime}\right)=\lim _{\lambda \rightarrow \infty} \limsup _{i \rightarrow \infty} F_{i}^{\lambda}(x) .
\end{aligned}
$$

It follows from Attouch's theorem that if $x \in \operatorname{dom} f$ is a point where each difference quotient $f_{x, h}(h>0)$ is proper and there exist $r \geq 0$ and $y_{0} \in X$ such that

$$
\inf _{h>0} f_{x, h}(y) \geq-r\left(\left\|y-y_{0}\right\|^{2}+1\right) \quad \forall y \in X,
$$

then the epi-derivative $f_{x}^{\prime}$ can be characterized as follows:

(III) $\lim _{\lambda \rightarrow \infty} \liminf _{h \downarrow 0} f_{x, h}^{\lambda}(y)=\lim _{\lambda \rightarrow \infty} \limsup _{h \downarrow 0} f_{x, h}^{\lambda}(y)=f_{x}^{\prime}(y) \quad \forall y \in X$.

Now suppose that $f$ is epi-differentiable at $x$. A vector $w \in X^{*}$ is called an epi-gradient of $f$ at $x$ if

$$
\langle w, y\rangle \leq f_{x}^{\prime}(y) \quad \forall y \in X .
$$

The set of vectors $w$ satisfying this condition is written $\partial f(x)$. For any $w$ in $\partial f(x)$, the second difference quotient of $f$ at $x$ relative to $w$ is the function $f_{x, w, h}: X \rightarrow \overline{\mathbb{R}}$ defined by

$$
f_{x, w, h}(y)=\frac{f(x+h y)-f(x)-h\langle w, y\rangle}{h^{2} / 2} .
$$

The function $f$ is called twice epi-differentiable at $x$ relative to $w$ with second epi-derivative $f_{x, w}^{\prime \prime}$ if the following equations hold with $f_{x, w}^{\prime \prime}(0)>-\infty$ :

$$
\limsup _{h \downarrow 0} \text { epi } f_{x, w, h}=\liminf _{h \downarrow 0} \operatorname{epi} f_{x, w, h}=\operatorname{epi} f_{x, w}^{\prime \prime} .
$$

Again, the first equation is the epi-differentiability criterion, while the second defines the function $f_{x, w}^{\prime \prime}$. There are alternative characterizations of $f_{x, w}^{\prime \prime}$, just as there are of $f_{x}^{\prime}$. 
The simplest interesting class of epi-differentiable functions is the set of convex functions. Rockafellar [12] proves the following results. Suppose $X=\mathbb{R}^{l}$ and $\sigma: X \rightarrow \mathbb{R}$ is convex and finite-valued. Then $\sigma$ is epi-differentiable at any point $\widehat{\alpha}$, where its epi-derivative coincides with the directional derivative

$$
\sigma_{\widehat{\alpha}}^{\prime}(\alpha)=\lim _{h \downarrow 0} \frac{\sigma(\widehat{\alpha}+h \alpha)-\sigma(\widehat{\alpha})}{h} .
$$

This is the support function of the set of epigradients $\partial \sigma(\widehat{\alpha})$, which agrees with the usual subdifferential of convex analysis. A sufficient condition for the convex function $\sigma$ to be twice epi-differentiable at $\widehat{\alpha}$ is that it be piecewise linearquadratic near $\widehat{\alpha}$, i.e., that some open cube centred at $\widehat{\alpha}$ admit a decomposition into finitely many polyhedral cells, in each of which $\sigma$ is either quadratic or affine. In this case any $\gamma$ in $\partial \sigma(\widehat{\alpha})$ obeys

$$
\sigma_{\widehat{\alpha}, \gamma}^{\prime \prime}(\alpha)=\sigma_{\widehat{\alpha}}^{\prime \prime}(\alpha)+\Psi_{\Sigma(\gamma)}(\alpha)
$$

where

$$
\begin{aligned}
\sigma_{\widehat{\alpha}}^{\prime \prime}(\alpha) & =\lim _{h \downarrow 0} \frac{\sigma(\widehat{\alpha}+h \alpha)-\sigma(\widehat{\alpha})-h \sigma_{\widehat{\alpha}}^{\prime}(\alpha)}{h^{2} / 2}, \\
\Sigma(\gamma) & =\left\{\alpha:\langle\alpha, \gamma\rangle=\sigma_{\widehat{\alpha}}^{\prime}(\alpha)\right\} .
\end{aligned}
$$

At the other extreme, consider a closed convex subset $C$ of $\mathbb{R}^{k}$, and the indicator function $\Psi_{C}: \mathbb{R}^{k} \rightarrow \overline{\mathbb{R}}$ defined by setting $\Psi_{C}(\alpha)=0$ if $\alpha \in C$, and $\Psi_{C}(\alpha)=+\infty$ otherwise. This function is also epi-differentiable at any point of its domain: given $\widehat{\alpha} \in C$, we have

$$
\left(\Psi_{C}\right)_{\widehat{\alpha}}^{\prime}(\alpha)=\Psi_{T_{C}(\widehat{\alpha})}(\alpha) \quad \forall \alpha \in \mathbb{R}^{k}
$$

where $T_{C}(\widehat{\alpha})$ is the usual closed convex cone of tangents to the set $C$ at the point $\widehat{\alpha}$. The epi-derivative is the support function of the normal cone (whose elements are row vectors)

$$
N_{C}(\widehat{\alpha})=\left\{\zeta \in\left(\mathbb{R}^{k}\right)^{*}:\langle\zeta, \alpha-\widehat{\alpha}\rangle \leq 0 \forall \alpha \in C\right\} .
$$

The second epiderivative of $\Psi_{C}$ is sure to exist if $C$ is polyhedral, i.e., the intersection of finitely many closed half-spaces.

After proving the preceding statements about convex functions, Rockafellar [12] generalizes them to sums and smooth compositions. As before, lê $\sigma: \mathbb{R}^{l} \rightarrow$ $\mathbb{R}$ be a finite-valued convex function, and let $C \subseteq \mathbb{R}^{k}$ be a closed convex set. Let $F: \mathbb{R}^{m} \rightarrow \mathbb{R}^{l}$ and $G: \mathbb{R}^{m} \rightarrow \mathbb{R}^{k}$ be continuously differentiable, and consider the function

$$
f(u)=\sigma(F(u))+\Psi_{C}(G(u)) .
$$

The domain of $f$ is the set $U=\left\{u \in \mathbb{R}^{m}: G(u) \in C\right\}$ : we call points in $U$ feasible. Associated with each feasible point $u$ is the following constraint qualification:

$$
\text { If } \eta \in N_{C}(G(u)) \text { and } \eta G^{\prime}(u)=0 \text {, then } \eta=0 \text {. }
$$

This is well known as the dual form of the Mangasarian-Fromovitz constraint qualification. It implies that the tangent cone to $U$ at $u$ is

$$
T_{U}(u)=\left\{v \in \mathbb{R}^{m}: G^{\prime}(u) v \in T_{C}(G(u))\right\} .
$$

We now quote Rockafellar [12, Theorem 4.5], calling on the notation of (4)(7). 
Theorem 2. If $u$ is feasible and satisfies $(C Q)$, then $f$ is both epi-differentiable and (Clarke-) subdifferentially regular at $u$, with

$$
f_{u}^{\prime}(v)=\sigma_{F(u)}^{\prime}\left(F^{\prime}(u) v\right)+\Psi_{T_{U}(u)}(v) .
$$

The function $f_{u}^{\prime}$ is the support function of the epigradient set

$$
\partial f(u)=\partial \sigma(F(u)) F^{\prime}(u)+N_{C}(G(u)) G^{\prime}(u) .
$$

If in addition $F$ and $G$ are $C^{2}, \sigma$ is piecewise linear quadratic, and $C$ is polyhedral, then $f$ is twice epi-differentiable at $u$ relative to any $w \in \partial f(u)$, with

$$
\text { (10) } f_{u, w}^{\prime \prime}(v)=\sigma_{F(u)}^{\prime \prime}\left(F^{\prime}(u) v\right)+\max _{(\gamma, \eta) \in \Gamma(u, w)}\left\{v^{T}[\gamma F+\eta G]^{\prime \prime}(u) v\right\}+\Psi_{\Sigma(u, w)}(v) \text {, }
$$

where

$$
\Gamma(u, w)=\left\{(\gamma, \eta) \in \partial \sigma(F(u)) \times N_{C}(G(u)): \gamma F^{\prime}(u)+\eta G^{\prime}(u)=w\right\}
$$

is a nonempty, bounded, polyhedral convex set, and

$$
\Sigma(u, w)=\left\{v \in \mathbb{R}^{m}: v \in T_{U}(u), \quad \sigma_{F(u)}^{\prime}\left(F^{\prime}(u) v\right)=\langle w, v\rangle\right\}
$$

is a polyhedral convex cone.

Note that the theorem's assertion of Clarke regularity implies that the epigradient set $\partial f(u)$ coincides with Clarke's generalized gradient of $f$ at $u$ (see [3]), which is given in the regular case as the set of (row vectors) $\zeta$ for which

$$
\liminf _{v \rightarrow u} \frac{f(v)-f(u)-\langle\zeta, v-u\rangle}{\|v-u\|} \geq 0
$$

\section{EPI-DeRIVATIVES OF INTEGRAL Functionals}

Consider the following integral functional defined on $L^{2}\left([0, T] ; \mathbb{R}^{m}\right)$ :

$$
\mathscr{I}(u)=\int_{0}^{T} f(t, u(t)) d t
$$

Here the integrand has the form $f(t, u)=\sigma(F(t, u))+\Psi_{C}(G(t, u))$, where the functions $F:[0, T] \times \mathbb{R}^{m} \rightarrow \mathbb{R}^{l}$ and $G:[0, T] \times \mathbb{R}^{m} \rightarrow \mathbb{R}^{k}$ are measurable in $t$ and continuously differentiable in $u$, the function $\sigma$ is convex and finitevalued on $\mathbb{R}^{l}$, and the set $C$ in $\mathbb{R}^{k}$ is nonempty, closed and convex. We write $U(t)=\left\{u \in \mathbb{R}^{m}: G(t, u) \in C\right\}$, and note that every $u$ in $L^{2}$ for which $\mathscr{I}(u)$ is finite must obey $u(t) \in U(t)$ almost everywhere. Throughout this section we deal with a fixed function $u$ in $L^{2}$ with $\mathscr{I}(u)$ finite. Our main results are Theorem 3, which deals with first-order derivatives, and Theorem 5, which treats the second-order case.

In the remainder of the paper, we reserve the notation $\langle\cdot, \cdot\rangle$ for the inner product in $L^{2}$; inner products in other spaces will be labelled explicitly, and finite-dimensional inner products will be indicated by simple juxtaposition. 
Theorem 3. Suppose there exist a constant $c \geq 0$ and an integrable function $\phi$ such that for almost all $t$ in $[0, T], u(t) \in U(t)$ satisfies both the constraint qualification $(C Q)$ and the growth condition

$$
\inf _{h>0} \frac{\sigma(F(u(t)+h x))-\sigma(F(u(t)))}{h} \geq \phi(t)-c|x|^{2} \quad \forall x \in \mathbb{R}^{m} .
$$

Then $\mathscr{I}$ is epi-differentiable at $u$, with epiderivative given by (see (8))

$$
\mathscr{I}_{u}^{\prime}(v)=\int_{0}^{T} f_{t, u(t)}^{\prime}(v(t)) d t \quad \forall v \in L^{2}
$$

The proof of Theorem 3 relies on some standard material from the theory of integral functionals. (See Rockafellar [11, Theorems 2A and 3A].) Consider a function $f:[0, T] \times \mathbb{R}^{m} \rightarrow \overline{\mathbb{R}}$ for which $u \mapsto f(t, u)$ is lower semicontinuous for each fixed $t$. Such an $f$ is called a normal integrand if the epigraph multifunction $t \Leftrightarrow$ epi $f(t, \cdot)$ is measurable. This is true if and only if $f$ itself is $\mathscr{L} \times \mathscr{B}$ measurable. If $f$ is a normal integrand for which the left side of equation (12) below is finite, then the equation itself is valid:

$$
\inf _{u \in L^{2}\left([0, T] ; \mathbb{R}^{m}\right)} \int_{0}^{T} f(t, u(t)) d t=\int_{0}^{T} \inf _{u \in \mathbb{R}^{m}} f(t, u) d t .
$$

Proof of Theorem 3. By hypothesis, the first difference quotient obeys the estimate below:

$$
\begin{aligned}
\mathscr{F}_{u, h}(v) & \geq \int_{0}^{T} \frac{\sigma(F(t, u(t)+h v(t)))-\sigma(F(t, u(t)))}{h} d t \\
& \geq \int_{0}^{T} \phi(t) d t-c\|v\|_{2}^{2} .
\end{aligned}
$$

Thus $\mathscr{J}_{u, h}$ is proper and condition (2) holds. By criterion (III) in Section 2, the epi-differentiability of $\mathscr{I}$ at $u$ is equivalent to

$$
\lim _{\lambda \rightarrow \infty} \liminf _{h \downarrow 0} \mathcal{F}_{u, h}^{\lambda}(v)=\lim _{\lambda \rightarrow \infty} \limsup _{h \downarrow 0} \mathscr{I}_{u, h}^{\lambda}(v) .
$$

For each $\lambda>0$, we have

$$
\begin{aligned}
\mathscr{I}_{u, h}^{\lambda}(v) & =\inf _{x \in L^{2}}\left\{\mathscr{I}_{u, h}(x)+\lambda\|x-v\|_{2}^{2}\right\} \\
& =\inf _{x \in L^{2}} \int_{0}^{T}\left[f_{t, u(t), h}(x(t))+\lambda|x(t)-v(t)|^{2}\right] d t .
\end{aligned}
$$

Now $\sigma$ and $\Psi_{C}$ are normal integrands, and $(t, x) \mapsto(F, G)(t, u(t)+h x)$ is measurable in $t$ and continuous in $x$, so the function $(t, x)$ $\mapsto f_{t, u(t), h}(x)$ is a normal integrand by [11, Proposition 2N]. Note that taking $x=0$ gives

$$
\inf _{x \in L^{2}} \int_{0}^{T}\left[f_{t, u(t), h}(x(t))+\lambda|x(t)-v(t)|^{2}\right] d t \leq \lambda \int_{0}^{T}|v(t)|^{2} d t<\infty .
$$

We may therefore apply (12) to take the infimum under the integral sign above and deduce that $\mathscr{F}_{u, h}^{\lambda}(v)=\int_{0}^{T} f_{t, u(t), h}^{\lambda}(v(t)) d t$. But

$$
f_{t, u(t), h}(x) \geq \phi(t)-c|x|^{2} \geq k_{1}(t)-2 c|x-v(t)|^{2},
$$


where $k_{1}(t):=\phi(t)-2 c|v(t)|^{2}$ is integrable. Thus whenever $\lambda \geq 2 c$ we have

$$
k_{1}(t) \leq f_{t, u(t), h}^{\lambda}(v(t))=\inf _{x \in \mathbb{R}^{m}}\left\{f_{t, u(t), h}(x)+\lambda|x-v(t)|^{2}\right\} \leq \lambda|v(t)|^{2} .
$$

By Fatou's lemma (assuming $\lambda \geq 2 c$ ) we have

$$
\liminf _{h \downarrow 0} \mathscr{F}_{u, h}^{\lambda}(v) \geq \int_{0}^{T} \liminf _{h \downarrow 0} f_{t, u(t), h}^{\lambda}(v(t)) d t .
$$

For any fixed $t$, the integrand on the right is nondecreasing as a function of $\lambda$, and is bounded below by $k_{1}(t)$. Thus the monotone convergence theorem applies, to give

$$
\lim _{\lambda \rightarrow \infty} \liminf _{h \downarrow 0} \mathcal{F}_{u, h}^{\lambda}(v) \geq \int_{0}^{T} \lim _{\lambda \rightarrow \infty} \liminf _{h \downarrow 0} f_{t, u(t), h}^{\lambda}(v(t)) d t=\int_{0}^{T} f_{u(t)}^{\prime}(v(t)) d t .
$$

Similarly, we have

$$
\lim _{\lambda \rightarrow \infty} \limsup _{h \downarrow 0} \mathcal{F}_{u, h}^{\lambda}(v) \leq \int_{0}^{T} \lim _{\lambda \rightarrow \infty} \limsup _{h \downarrow 0} f_{t, u(t), h}^{\lambda}(v(t)) d t=\int_{0}^{T} f_{u(t)}^{\prime}(v(t)) d t .
$$

Combining these two inequalities confirms $(\ddagger)$.

Here is an immediate consequence of Theorem 3 in terms of epigradients.

Corollary 4. Under the conditions of 3 , the set of epigradients of $\mathscr{I}$ at $u$ is

$$
\partial \mathscr{I}(u)=\left\{w \in L^{2}: w(t) \in \partial f(t, u(t)) \text { a.e. } t \in[0, T]\right\} \text {. }
$$

Here $\partial f(t, u(t))$ refers to the set of epigradients in $u$ for fixed $t$; see (9). Proof. If $w \in L^{2}$ satisfies $w(t) \in \partial f(t, u(t))$ a.e., then we have

$$
\mathscr{J}_{u}^{\prime}(v)=\int_{0}^{T} f_{t, u(t)}^{\prime}(v(t)) d t \geq \int_{0}^{T} w(t) v(t) d t=\langle w, v\rangle
$$

for any $v$ in $L^{2}$, so $w$ lies in $\partial \mathscr{I}(u)$.

On the other hand, if $w$ belongs to $\partial \mathscr{I}(u)$, then for any $v \in L^{2}$ we have

$$
\langle w, v\rangle \leq \mathscr{I}_{u}^{\prime}(v)=\int_{0}^{T} f_{t, u(t)}^{\prime}(v(t)) d t
$$

that is,

$$
\inf _{v \in L^{2}} \int_{0}^{T}\left[f_{t, u(t)}^{\prime}(v(t))-w(t) v(t)\right] d t \geq 0 .
$$

Now $(f)_{t, u(t)}^{\prime}(x)=g_{(F, G)(t, u(t))}^{\prime}\left((F, G)^{\prime}(t, u(t)) x\right)=\lim _{h \downarrow 0} g_{h}(t, x)$, where

$$
g_{h}(t, x)=\frac{\sigma(F(t, u(t)+h x))-\sigma(F(t, u(t)))+\Psi_{C}(G(t, u(t)+h x))}{h} .
$$

Since $g_{h}$ is a normal integrand, it must be $\mathscr{L} \times \mathscr{B}$ measurable. Therefore $(f)_{t, u(t)}^{\prime}(x)$, being the pointwise limit of $g_{h}(t, x)$, must also be $\mathscr{L} \times \mathscr{B}$ measurable. It is also lower semicontinuous with respect to $x$ by the definition of the epi-derivative. Thus $(t, x) \mapsto(f)_{t, u(t)}^{\prime}(x)$ is a normal integrand. By applying (12) to $(*)$ we get

$$
\int_{0}^{T} \inf _{v \in \mathbb{R}^{m}}\left[f_{t, u(t)}^{\prime}(v)-w(t) v\right] d t \geq 0
$$


Since the integrand here is obviously nonpositive (take $v=0$ ), it must actually vanish for almost all $t$. That is, $w(t) \in \partial f(t, u(t))$ almost everywhere.

Now we turn to the second order epi-differentiability of $\mathscr{I}$.

Theorem 5. Suppose both functions $F$ and $G$ are $C^{2}$ in $x$, the convex function $\sigma$ is piecewise linear quadratic, and the set $C$ is polyhedral. Suppose further that there exist $c \geq 0$ and $\phi \in L^{1}[0, T]$ such that for almost all $t$ in $[0, T]$, the set $U(t)$ is convex, the vector $u(t) \in U(t)$ satisfies the constraint qualification $(C Q)$, and for any $\gamma$ in $\partial \sigma(F(t, u(t)))$ one has the growth condition

$$
\inf _{h>0} \frac{\sigma(F(t, u(t)+h x))-\sigma(F(t, u(t)))-h \gamma F^{\prime}(t, u(t)) x}{h^{2} / 2} \geq \phi(t)-c|x|^{2}
$$

$\forall x \in \mathbb{R}^{m}$.

Then $\mathscr{I}$ is twice epi-differentiable at $u$. Its second epi-derivative relative to $w$ in $\partial \mathscr{I}(u)$ is (see (10))

$$
\mathscr{J}_{u, w}^{\prime \prime}(v)=\int_{0}^{T} f_{t, u(t), w(t)}^{\prime \prime}(v(t)) d t \quad \forall v \in L^{2}
$$

Proof. Since $w(t)$ is an epi-gradient of $f$ at $u(t)$, Theorem 2 provides $\gamma(t) \in$ $\partial \sigma(F(t, u(t)))$ and $\eta(t) \in N_{C}(G(t, u(t)))$ such that

$$
w(t)=\gamma(t) F^{\prime}(t, u(t))+\eta(t) G^{\prime}(t, u(t))
$$

Fix $x$ in $\mathbb{R}^{m}$. If $u(t)+h x \notin U(t)$, then $f_{t, u(t), w(t), h}(x)=\infty$. Otherwise, the convexity of $U(t)$ implies $x \in T_{U(t)}(u(t))$, that is, $G^{\prime}(t, u(t)) x \in$ $T_{C}(G(t, u(t)))$, so $\eta(t) G^{\prime}(t, u(t)) x \leq 0$. Thus our assumed growth condition leads to the estimate

$$
\begin{aligned}
& f(t, u(t)+h x)-f(t, u(t))-h w(t) x \\
&= \sigma(F(t, u(t)+h x))-\sigma(F(t, u(t)))-h w(t) x \\
&= \sigma(F(t, u(t)+h x))-\sigma(F(t, u(t))) \\
& \quad-h \gamma(t) F^{\prime}(t, u(t)) x-h \eta(t) G^{\prime}(t, u(t)) x \\
& \geq\left(\phi(t)-c|x|^{2}\right) h^{2} / 2 .
\end{aligned}
$$

In either case, we have $f_{t, u(t), w(t), h}(x) \geq \phi(t)-c|x|^{2}$ for all $x$. Thus for any $0<h<1$ and $v \in L^{2}$, the second difference quotient $\mathscr{I}_{u, w, h}(v)=$ $\int_{0}^{T} f_{t, u(t), w(t), h}(v(t)) d t$ is at least $\int_{0}^{T} \phi(t) d t-c\|v\|_{2}^{2}$, which confirms the growth condition (2) of Attouch's theorem. By the second-order cognate of criterion (III) in Section 2, the second-order epi-differentiability of $\mathscr{I}$ at $u$ relative to $w$ is equivalent to

$$
\lim _{\lambda \rightarrow \infty} \liminf _{h \downarrow 0} \mathcal{F}_{u, w, h}^{\lambda}(v)=\lim _{\lambda \rightarrow \infty} \limsup _{h \downarrow 0} \mathscr{I}_{u, w, h}^{\lambda}(v) .
$$

For each $\lambda>0$, we have

$$
\begin{aligned}
\mathscr{F}_{u, w, h}^{\lambda}(v) & =\inf _{x \in L^{2}}\left\{\mathscr{J}_{u, w, h}(x)+\lambda\|x-v\|_{2}^{2}\right\} \\
& =\inf _{x \in L^{2}} \int_{0}^{T}\left(f_{t, u(t), w(t), h}(x(t))+\lambda|x(t)-v(t)|^{2}\right) d t .
\end{aligned}
$$


Since $f_{t, u(t), w(t), h}(x)$ is a normal integrand and $f_{t, u(t), w(t), h}(x) \geq \phi(t)-$ $2 c|v(t)|^{2}-2 c|x-v(t)|^{2}$, we also have

$$
k_{1}(t):=\phi(t)-2 c|v(t)|^{2} \leq f_{t, u(t), w(t), h}^{\lambda}(v(t)) \leq \lambda|v(t)|^{2}
$$

for $\lambda \geq 2 c$. Thanks to Fatou's lemma and the monotone convergence theorem (see the proof of Theorem 3), the result follows.

Let us apply our differentiation theorem to the integrand

$$
f(t, u)=\max _{1 \leq i \leq l_{1}}\left\{f_{i}(t, u)\right\}+\sum_{i=l_{1}+1}^{l} \rho_{i}\left(d_{i}\left(f_{i}(t, u)\right)\right)+\Psi_{C}(G(t, u)) .
$$

Here each $\rho_{i}:[0, \infty) \rightarrow \mathbb{R}$ has the form $\rho_{i}(x)=\frac{1}{2} r_{i} x^{2}+a_{i} x+b_{i}$ with $r_{i} \geq 0$ and $a_{i} \geq 0$. So $\rho_{i}$ is a nondecreasing affine $\left(r_{i}=0\right)$ or convex quadratic $\left(r_{i}>0\right)$ function. Each $d_{i}$ is a distance function associated with either an infinite interval or a single point:

$$
d_{i}(z)= \begin{cases}\max \left\{0, z-c_{i}\right\}, & \text { if } i=l_{1}+1, \ldots, l_{2}, \\ \left|z-c_{i}\right|, & \text { if } i=l_{2}+1, \ldots, l\end{cases}
$$

Thus $f$ can be intepreted as a maximum of finitely many smooth functions, plus a standard augmented penalty function (optional-set $\rho_{i} \equiv 0$ ), plus an infinite penalty function (optional-choose $C=\mathbb{R}^{m}$ ).

We express the integrand in the simple form

$$
f(t, u)=\sigma(F(t, u))+\Psi_{C}(G(t, u))
$$

by introducing the notation

$$
\begin{aligned}
\sigma(\alpha) & =\max _{1 \leq i \leq l_{1}}\left\{\alpha_{i}\right\}+\sum_{i=l_{1}+1}^{l} \rho_{i}\left(d_{i}\left(\alpha_{i}\right)\right), \\
F(t, u) & =\left(f_{1}(t, u), \ldots, f_{l}(t, u)\right) .
\end{aligned}
$$

We also denote

$$
\begin{aligned}
I(t, u) & =\left\{1 \leq j \leq l_{1}: f_{j}(t, u)=\max _{1 \leq i \leq l_{1}} f_{i}(t, u)\right\}, \\
S(t, u) & =\left\{\gamma \in \mathbb{R}_{+}^{l_{1}}: \sum_{i=1}^{l_{1}} \gamma_{i}=1 ; \gamma_{i}=0, i \notin I(t, u)\right\}, \\
p_{i}(t, u) & =\rho_{i}^{\prime}\left(d_{i}\left(f_{i}(t, u)\right)\right)=r_{i} d_{i}\left(f_{i}(t, u)\right)+a_{i}, \\
S_{i}(t, u) & =p_{i}(t, u) \partial d_{i}\left(f_{i}(t, u)\right) \text { for } i=l_{1}+1, \ldots, l .
\end{aligned}
$$

In this notation, the epiderivative and subgradient of the convex function $\sigma$ are given by

$$
\begin{aligned}
\sigma_{F(t, u)}^{\prime}(\alpha) & =\max _{j \in I(t, u)}\left\{\alpha_{j}\right\}+\sum_{i=l_{1}+1}^{l} p_{i}(t, u)\left(d_{i}\right)_{f_{i}(t, u)}^{\prime}\left(\alpha_{i}\right) \\
\partial \sigma(F(t, u)) & =S(t, u) \times S_{l_{1}+1}(t, u) \times \cdots \times S_{l}(t, u) .
\end{aligned}
$$


According to Theorem 2, the function $f(t, \cdot)$ is twice epi-differentiable at any point $u \in U(t)$ where the constraint qualification $(C Q)$ holds. Its first epiderivative with respect to $u$ is given by

$$
f_{t, u}^{\prime}(v)=\sigma_{F(t, u)}^{\prime}\left(F^{\prime}(t, u) v\right)+\Psi_{T_{U}(u)}(v) .
$$

Its second epi-derivative in $u$, relative to some $w \in \partial f(t, u)$, is given by

$$
\begin{aligned}
f_{t, u, w}^{\prime \prime}(v)= & \sum_{i=l_{1}+1}^{l} \\
& r_{i}\left[\left(d_{i}\right)_{f_{i}(t, u)}^{\prime}\left(f_{i}^{\prime}(t, u)^{T} v\right)\right]^{2} \\
& +\max _{(\gamma, \eta) \in \Gamma(t, u, w)}\left\{v^{T}[\gamma F+\eta G]^{\prime \prime}(t, u) v\right\}+\Psi_{\Sigma(t, u, w)}(v),
\end{aligned}
$$

where

$$
\begin{aligned}
& \Gamma(t, u, w)=\left\{(\gamma, \eta) \in \partial \sigma(F(t, u)) \times N_{C}(G(t, u)):\right. \\
& \left.\gamma F^{\prime}(t, u)+\eta G^{\prime}(t, u)=w\right\} \\
& \Sigma(t, u, w)=\left\{v \in T_{U(t)}(u): \sigma_{F(t, u)}^{\prime}\left(F^{\prime}(t, u) v\right)+\Psi_{T_{U}(u)}(v)=w v\right\} .
\end{aligned}
$$

These finite-dimensional calculations underlie the following infinite-dimensional result.

Theorem 6. With $f$ defined in (14), let $u$ be a function such that $u(t) \in U(t)$ satisfies (CQ) for almost all $t$. Suppose there exists a constant $c \geq 0$ such that for almost all $t$, any $x$ and $y$ in $\mathbb{R}^{m}$ satisfy

(i) $y^{T} f_{i}^{\prime \prime}(t, x) y \geq-c|y|^{2}$ for $i=1, \ldots, l_{2}$,

(ii) $\left|y^{T} \cdot f_{i}^{\prime \prime}(t, x) y\right| \leq c|y|^{2}$ for $i=l_{2}+1, \ldots, l$,

(iii) $p_{i}(t, u(t)) \leq c$ for $i=l_{1}+1, \ldots, l$.

Suppose further that $\left|F^{\prime}(t, u(t))\right|^{2}$ is integrable on $[0, T]$. Then $\mathscr{I}$ is epidifferentiable at $u$, and its epi-derivative is given by $\mathscr{I}_{u}^{\prime}(v)=\int_{0}^{T} f_{t, u(t)}^{\prime}(v(t)) d t$. Furthermore, if $U(t)$ is convex, then $\mathscr{I}$ is twice epi-differentiable at $u$ and its second epi-derivative relative to any $w \in \partial \mathscr{F}(u)$ is given by $\mathscr{I}_{u, w}^{\prime \prime}(v)=$ $\int_{0}^{T} f_{t, u(t), w(t)}^{\prime \prime}(v(t)) d t$.

Proof. The conclusions are precisely those of Theorems 3 and 5: we need only establish the growth conditions of those two results. The arguments in both cases are similar, so we discuss only the more demanding second-order condition of Theorem 5. For any $\gamma \in \partial \sigma(F(t, u(t)))$, the definition of subgradient and the mean value theorem imply

$$
\begin{aligned}
\frac{\sigma(F(u(t)+h x))-\sigma(F(u(t)))}{h^{2} / 2} & \geq \gamma\left(\frac{F(t, u(t)+h x)-F(t, u(t))}{h^{2} / 2}\right) \\
& =\gamma\left(\frac{h F^{\prime}(t, u(t)) x}{h^{2} / 2}+F^{\prime \prime}(t, \widetilde{u})(x, x)\right) .
\end{aligned}
$$

Now $\gamma \in \partial \sigma(F(t, u(t)))$, so $0 \leq \gamma_{i} \leq 1$ for $i=1, \ldots, l_{1}$, and $0 \leq \gamma_{i} \leq$ $p_{i}(t, u(t)) \leq c$ for $i=l_{1}+1, \ldots, l_{2}$, and $\left|\gamma_{i}\right| \leq p_{i}(t, u(t)) \leq c$ for $i=$ 
$l_{2}+1, \ldots, l$. Thus our previous estimate implies

$$
\begin{aligned}
& \frac{\sigma(F(t, u(t)+h x))-\sigma(F(t, u(t)))-h \gamma F^{\prime}(t, u(t)) x}{h^{2} / 2} \\
& \geq-\sum_{i=1}^{l_{1}} c \gamma_{i}|x|^{2}-\sum_{i=l_{1}+1}^{l} c p_{i}(t, u(t))|x|^{2} \\
& \geq-\widetilde{c}|x|^{2}
\end{aligned}
$$

for some constant $\tilde{c}$. This establishes the growth condition of Theorem 5, and hence the result.

Comparison to other work. The generalization of epi-convergence from finite to infinite-dimensional spaces calls for some discussion of the topologies involved. Mosco epi-convergence, defined by changing " $y_{i} \rightarrow y$ " to " $y_{i}$ converges weakly to $y$ " in criterion $\mathrm{I}(\mathrm{i})$ of Section 2 , is appropriate in many problems, including the analysis of convex integral functionals-see Do [5]. However, the Mosco epi-limit of any functional is, by definition, weakly lower semicontinuous, and it is well known that every weakly lower semicontinuous integral functional is convex. Thus our problem of treating nonconvex integrands, and obtaining correspondingly nonconvex results, can be solved only in some stronger topology.

Cominetti's work on general amenable functions [4] on reflexive Banach spaces deals exclusively with epidifferentiability in the Mosco sense. Thus, although [4, Theorem 4.4, page 860] and our Theorem 5 look similar, the former does not give useful second-order information for the integral functionals considered here. (The problem can be traced to the weak-continuity assumption on the second-derivative mapping in [4, Theorem 4.4].) We note, however, that Cominetti's Theorem 4.4 has other uses, and that in particular its finitedimensional instance generalizes Theorem 2 of this paper.

Noll [8] discusses the (strong) epi-differentiability of integral functionals whose integrands are finite and have second-order Taylor expansions. Thus his integrands are at least Fréchet differentiable. He shows that in this case, the second epi-derivative is a quadratic functional [8, Theorem 3.1]. In contrast, we deal with extended real-valued, nonsmooth integrands. Our results include the case where $f(t, u)=F(t, u)$ is smooth and scalar-valued as a function of $u$ : we simply choose $l=1, \sigma(\alpha)=\alpha, G \equiv 0$, and $C=\mathbb{R}^{k}$. In this case $\partial f(t, u)=\left\{F^{\prime}(t, u)\right\}$, and it is easy to calculate $\Gamma(t, u, w)=\{(1,0)\}$ and $\Sigma(t, u, w)=\mathbb{R}$, so $f_{t, u, w}^{\prime \prime}(v)=v^{T} F^{\prime \prime}(t, u) v$. If we add the assumption that $\inf _{x \in \mathbb{R}^{m}} F^{\prime \prime}(t, x)>-\infty$, then our Theorem 3 shows that $\mathscr{I}$ is twice epi-differentiable at $u$ with second epi-derivative

$$
\mathscr{I}_{u, w}^{\prime \prime}(v)=\int_{0}^{T} v^{T}(t) F^{\prime \prime}(t, u(t)) v(t) d t
$$

as expected. Of course, membership in the class $C^{2}$ is a stronger condition on $F$ than the existence of a second-order Taylor expansion. But even in this case, we have something Noll [8] does not cover. He considers only integrands bounded by quadratic functions $[8,(3.2)]$, or, in the $C^{2}$ case, integrands whose Hessian matrices are bounded [8, Theorem 4.2]. We only impose a lower bound on the Hessian matrix. Thus our theory pertains to arbitrary smooth convex integrands (such as $f(t, u)=e^{u}$ in the case $m=1$ ), whereas Noll's requires 
that the integrand grow at most quadratically. As for functionals in Sobolev spaces, or in our terms Bolza functionals, a similar remark applies.

Levy [6] discusses the strong epi-differentiability of integral functionals in $L^{p}$ spaces and applies the results to the sensitivity analysis of set-valued functions. His paper contains results analogous to Theorems 3 and 5, which we proved independently at about the same time. (The proof techniques, however, are different: Levy chooses sequences satisfing criterion (I) directly, whereas we pass to Moreau-Yosida approximates and prove (III).) Indeed, a draft of [6] arrived in time for us to state Theorem 3 and Theorem 5 in a form that facilitates direct comparison. Our Theorem 6 , which identifies a significant class of functions to which the general theory applies, has no counterpart in [6]. Finally, our applications are disjoint from those in [6]: whereas Levy concentrates on sensitivity analysis, we discuss the epi-differentiability of Bolza functionals and the resulting necessary conditions in optimal control.

\section{Bolza FUnCTIONALS}

Consider the functional $\mathscr{J}: L^{2}\left([0, T], \mathbb{R}^{m}\right) \rightarrow \overline{\mathbb{R}}$ defined by

$$
\mathscr{J}(u)=\int_{0}^{T}\left[L(t, x(t), u(t))+\Psi_{U(t)}(u(t))\right] d t,
$$

where $U(t)=\left\{u \in \mathbb{R}^{m}: G(t, u) \in C\right\}$ as before, and the function $x$ is given in terms of $u$ by $x(t)=a(t)+E(u)(t)$ for some operator $E \in \mathscr{L}\left(L^{2}\left([0, T] ; \mathbb{R}^{m}\right)\right.$, $\left.L^{2}\left([0, T] ; \mathbb{R}^{n}\right)\right)$ and function $a \in L^{2}$. Note that the choices $m=n$ and $E(u)(t)=x_{0}+\int_{0}^{t} u(r) d r$ imply $\dot{x}=u$ a.e., so that $\mathscr{J}$ takes the form of a classical Bolza functional with an infinite penalty for derivative values $\dot{x}(t)$ outside the prescribed velocity set $U(t)$. In this section we study the epi-differentiability of $\mathscr{J}$.

As in Section 3, we assume that $C$ is a closed convex subset of $\mathbb{R}^{k}$, while $G(t, u):[0, T] \times \mathbb{R}^{m} \rightarrow \mathbb{R}^{k}$ is measurable in $t$ and $C^{2}$ in $u$. The integrand $L$ must satisfy the following conditions:

(1) The function $L(t, x, u)$ is measurable in $t$ and $C^{2}$ in $(x, u)$;

(2) Both $\int_{0}^{T}|L(t, 0,0)|^{2} d t<\infty$ and $\int_{0}^{T}\left|L^{\prime}(t, 0,0)\right|^{2} d t<\infty$;

(3) There exists a constant $c \geq 0$ such that $\left|L^{\prime \prime}(t, x, u)\right| \leq c$ for all $(t, x, u)$ in $[0, T] \times \mathbb{R}^{n+m}$.

(In (2) and (3), the gradient $L^{\prime}$ and Hessian $L^{\prime \prime}$ refer to the vector argument $(x, u)$ in $\mathbb{R}^{n+m}$.)

To differentiate $\mathscr{J}$, we first decompose $\mathscr{J}=\mathscr{I}(\Phi(u))$, where $\mathscr{I}(u, v)=$ $\mathscr{I}_{1}(x, u)+\mathscr{I}_{2}(u)$ is the functional on $L^{2} \times L^{2}$ defined in terms of

$$
\begin{aligned}
\mathscr{I}_{1}(x, u) & =\int_{0}^{T} L(t, x(t), u(t)) d t, \\
\mathscr{F}_{2}(u) & =\int_{0}^{T} \Psi_{U(t)}(u(t)) d t, \\
\Phi(u) & =(a+E(u), u) .
\end{aligned}
$$

Note that the functional $\mathscr{I}_{1}$ is continuously (Fréchet) differentiable and twice weakly Gâteaux differentiable, but in general it is not twice continuously differentiable unless $L$ is precisely a quadratic function. (See Noll [8] for a detailed example and further references.) 
Since $\mathscr{I}_{1} \circ \Phi \in C^{1}$, we have $\partial \mathscr{J}(u)=\mathscr{I}_{1}^{\prime}(\Phi(u)) \Phi^{\prime}(u)+\partial \mathscr{I}_{2}(u)$. Thus every $w \in \partial \mathscr{J}(u)$ gives rise to a functional $w_{1}:=w-\mathscr{J}_{1}^{\prime}(\Phi(u)) \Phi^{\prime}(u)$ in $\partial \mathscr{F}_{2}(u)$, so that $w_{1}(t) \in N_{U(t)}(u(t))$ a.e. by Corollary 4. For any $v \in L^{2}$, $\Phi^{\prime}(u) v=(E(v), v)$ : writing $y=E(v)$ gives

$$
\begin{aligned}
\langle w, v\rangle & =\left\langle\mathscr{I}_{1}^{\prime}(\Phi(u)) \Phi^{\prime}(u), v\right\rangle+\left\langle w_{1}, v\right\rangle \\
& =\left\langle\mathcal{F}_{1}^{\prime}(\Phi(u)), \Phi^{\prime}(u) v\right\rangle_{L^{2} \times L^{2}}+\left\langle w_{1}, v\right\rangle \\
& =\int_{0}^{T}\left[L_{x}(t) y(t)+L_{u}(t) v(t)+w_{1}(t) v(t)\right] d t \\
& =\left\langle\left(L_{x}, L_{u}+w_{1}\right), \Phi^{\prime}(u) v\right\rangle_{L^{2} \times L^{2}} .
\end{aligned}
$$

So we have $w=\Phi^{\prime}(u)^{*} \bar{w}$, where $\bar{w}(t)=\left(L_{x}(t), L_{u}(t)+w_{1}(t)\right)$.

We now describe the epi-differentiability of $\mathscr{J}$.

Theorem 7. If the constraint qualification $(C Q)$ holds at $u(t)$ for almost all $t$, then $\mathcal{J}$ is epi-differentiable at $u$. Its epi-derivative is given by

$$
\mathscr{J}_{u}^{\prime}(v)=\mathscr{I}_{(x, u)}^{\prime}(y, v)=\int_{0}^{T}\left[L_{x}(t) y(t)+L_{u}(t) v(t)+\Psi_{T_{U(t)}}(u(t))\right] d t,
$$

where $y=E(v)$. If, in addition, the set $U(t)$ is convex, then $\mathscr{J}$ is twice epi-differentiable at $u$ relative to $w \in \partial \mathscr{J}(u)$, and its second epi-derivative is

$$
\begin{aligned}
\mathscr{J}_{u, w}^{\prime \prime}(v)=\int_{0}^{T}\left[L_{2}(\right. & t, v(t), y(t)) \\
& \left.\quad+\max _{\eta \in \Gamma(t)}\left\{v(t)^{T}\left(\eta^{T} G\right)^{\prime \prime}(t, u(t)) v(t)\right\}+\Psi_{\Sigma(t)}(v(t))\right] d t .
\end{aligned}
$$

Here $w_{1}:=w-\mathcal{F}_{1}^{\prime}(\Phi(u)) \Phi^{\prime}(u)$ and

$$
\begin{aligned}
L_{2}(t, v, y) & =v^{T} L_{u u}(t) v+2 y^{T} L_{u x}(t) v+y^{T} L_{x x}(t) y, \\
\Gamma(t) & =\left\{\eta \in N_{C}(G(t, u(t))): \eta G^{\prime}(t, u(t))=w_{1}(t)\right\}, \\
\Sigma(t) & =\left\{v \in T_{U(t)}(u(t)): w_{1}(t) v=0\right\} .
\end{aligned}
$$

Proof. Note that $\mathscr{I}=\mathscr{I}_{1}+\mathscr{I}_{2}$ is epi-differentiable, since $\mathscr{I}_{1}$ is continuously differentiable and $\mathscr{I}_{2}$ is epi-differentiable by Theorem 3. This is relevant because $\mathscr{J}_{u, h}(v)=\mathscr{I}_{(x, u), h}(y, v)$ where $y=E(v)$. By criterion (I) in Section 2, our first-order assertions regarding $\mathscr{J}$ will follow if for any point $v \in L^{2}$ and any sequence $h_{i} \downarrow 0$, both

(i) for any sequence $v_{i}$ converging to $v$, we have $\liminf _{i \rightarrow \infty} \mathscr{J}_{u}, h_{i}\left(v_{i}\right) \geq$ $\mathcal{I}_{(u, x)}^{\prime}(v, y)$; and

(ii) there exists a sequence $v_{i}$ converging to $v$, such that $\lim _{\sup } \rightarrow \infty \mathscr{J}_{u}, h_{i}\left(v_{i}\right)$ $\leq \mathscr{I}_{(u, x)}^{\prime}(v, y)$.

Given any sequence $v_{i} \rightarrow v$ in $L^{2}$, let $y_{i}=E\left(v_{i}\right)$. Then $\left(v_{i}, y_{i}\right) \rightarrow(v, y)$ in $L^{2} \times L^{2}$. Since $\mathscr{I}$ is epi-differentiable at $(u, x)$, we have

$$
\liminf _{i \rightarrow \infty} \mathscr{F}_{(x, u), h_{i}}\left(y_{i}, v_{i}\right) \geq \mathcal{F}_{(x, u)}^{\prime}(y, v),
$$

so condition (i) holds. It remains to prove (ii). This is trivial if $\mathscr{F}_{(x, u)}^{\prime}(y, v)=$ $\infty$, so we assume that $\mathscr{I}_{(u, x)}^{\prime}(v, y)$ is finite. The epi-differentiability of $\mathscr{I}$ implies that there exists a sequence $\left(w_{i}, v_{i}\right) \rightarrow(y, v)$ such that

$$
\limsup _{i \rightarrow \infty} \mathcal{F}_{(x, u), h_{i}}\left(w_{i}, v_{i}\right) \leq \mathcal{F}_{(x, u)}^{\prime}(y, v) .
$$


Since the right side is finite, we have $u(t)+h_{i} v_{i}(t) \in U(t)$ almost everywhere for $i$ sufficiently large. Now by definition,

$$
\mathscr{I}_{(x, u), h_{i}}\left(w_{i}, v_{i}\right)=\mathscr{I}_{(x, u), h_{i}}\left(y_{i}, v_{i}\right)+\epsilon_{i}
$$

where

$$
\epsilon_{i}=\int_{0}^{T} \frac{L\left(t, x+h_{i} w_{i}, u+h_{i} v_{i}\right)-L\left(t, x+h_{i} y_{i}, u+h_{i} v_{i}\right)}{h_{i}} d t
$$

Using the mean value theorem, we estimate

$$
\begin{aligned}
\mid L(t, & x+y, u+v)-L(t, x+z, u+v) \mid \\
& =\left|L_{x}(t, x+\theta y+(1-\theta) z, u+v)\right||y-z| \\
& \leq\left(\left|L_{x}(t, x, u)\right|+c|y|+c|z|+c|v|\right)|y-z| .
\end{aligned}
$$

It follows that

$$
\left|\epsilon_{i}\right| \leq \int_{0}^{T}\left(\left|L_{x}(t, x, u)\right|+c\left|w_{i}\right|+c\left|y_{i}\right|+c\left|v_{i}\right|\right)\left|w_{i}-y_{i}\right| d t .
$$

The sequence of integrals $\int_{0}^{T}\left(\left|L_{x}(t, x(t), u(t))\right|+c\left|v_{i}(t)\right|+c\left|w_{i}(t)\right|+c\left|y_{i}(t)\right|\right)^{2} d t$ is bounded and $\int_{0}^{T}\left|w_{i}(t)-y_{i}(t)\right|^{2} d t \rightarrow 0$. Therefore $\epsilon_{i} \rightarrow 0$ as $i \rightarrow \infty$. So

$$
\limsup _{i \rightarrow \infty} \mathscr{J}_{u, h_{i}}\left(y_{i}\right)=\limsup _{i \rightarrow \infty} \mathscr{I}_{(x, u), h_{i}}\left(w_{i}, v_{i}\right) \leq \mathscr{I}_{(x, u)}^{\prime}(y, v) .
$$

By criterion (I), the functional $\mathcal{J}$ is epi-differentiable at $u$ with epi-derivative $\mathcal{F}_{(u, x)}^{\prime}(v, y)$.

Next, suppose $w \in \partial \mathscr{J}(u)$. The second difference quotient of $\mathscr{J}$ at $u$ relative to $w$ is

$$
\mathscr{J}_{u, w, h}(v)=\mathscr{I}_{(x, u),\left(L_{x}, L_{u}+w_{1}\right), h}(y, v) .
$$

Note that $\mathscr{I}$ is twice epi-differentiable at $(x, u)$ relative to $\left(L_{x}, L_{u}+w_{1}\right)$, so a discussion similar to that for the first epi-derivative shows that $\mathcal{J}$ is twice epi-differentiable at $u$. The only difference is the estimate of $\epsilon_{i}$. This time $\epsilon_{i}$ equals

$$
\int_{0}^{T} \frac{L\left(t, x+h_{i} w_{i}, u+h_{i} v_{i}\right)-L\left(t, x+h_{i} y_{i}, u+h_{i} v_{i}\right)-h_{i} L_{x}(t, x, u)\left(w_{i}-y_{i}\right)}{h_{i}^{2} / 2} d t .
$$

The mean value theorem provides the estimate

$$
\begin{aligned}
\mid L(t, x+y & , u+v)-L(t, x+z, u+v)-L_{x}(t, x, u)(y-z) \mid \\
& =\left|\left(L_{x}(t, x+\theta y+(1-\theta) z, u+v)-L_{x}(t, x, u)\right)(y-z)\right| \\
& \leq|y-z|(c|y|+c|z|+c|v|),
\end{aligned}
$$

which implies that

$$
\left|\epsilon_{i}\right| \leq \int_{0}^{T} 2 c\left(\left|w_{i}(t)\right|+\left|y_{i}(t)\right|+\left|v_{i}(t)\right|\right)\left|w_{i}(t)-v_{i}(t)\right| d t .
$$

Consequently $\epsilon_{i} \rightarrow 0$ as $i \rightarrow \infty$, as required. 


\section{AN OPTIMAL CONTROL PROBLEM}

In this section, we apply our results to obtain necessary conditions for optimality in the following free endpoint control problem:

$$
\begin{array}{ll}
\text { minimize } & \int_{0}^{T} L(t, x(t), u(t)) d t, \\
\text { subject to } & \dot{x}(t)=A(t) x(t)+B(t) u(t) \text { a.e., } x(0)=x_{0}, \\
& u(t) \in U(t) \text { a.e. }
\end{array}
$$

Here the state $x$ evolves in $\mathbb{R}^{n}$ and the control $u$ takes values in $\mathbb{R}^{m}$, so the measurable matrix-valued functions $A$ and $B$ have dimensions $n \times n$ and $n \times m$ respectively. As before, the control set is $U(t)=\left\{u \in \mathbb{R}^{m}: G(t, u) \in C\right\}$.

Let $X$ be the fundamental matrix function associated with $A$, i.e., the unique solution of the initial-value problem $X^{\prime}(t)=A(t) X(t), X(0)=I$. Using $X$, we define the operator $E: L^{2}\left([0, T] ; \mathbb{R}^{m}\right) \rightarrow L^{2}\left([0, T] ; \mathbb{R}^{n}\right)$ and the function $a \in L^{2}\left([0, T] ; \mathbb{R}^{n}\right)$ by

$$
E(u)(t)=\int_{0}^{t} X(t) X(s)^{-1} B(s) u(s) d s, \quad a(t)=X(t) x_{0} .
$$

Then the controlled dynamics above reduce to the equation $x=a+E(u)$, so problem $(P)$ takes the form

$$
\operatorname{minimize} \mathscr{J}(u)=\int_{0}^{T}\left[L(t, a(t)+E(u)(t), u(t))+\Psi_{U(t)}(u(t))\right] d t .
$$

We will apply the analysis of $\mathscr{J}$ in Section 4 to derive optimality conditions for $(P)$.

The pre-Hamiltonian for problem $(P)$ is

$$
H(t, x, p, u)=p^{T}(A(t) x+B(t) u)-L(t, x, u) .
$$

We also consider the extended pre-Hamiltonian function below, which incorporates the control constraints through a multiplier vector $\eta \in\left(\mathbb{R}^{k}\right)^{*}$ :

$$
\mathscr{H}(t, x, p, u, \eta)=p^{T}(A(t) x+B(t) u)-L(t, u, x)-\sum_{i=1}^{k} \eta_{i} g_{i}(t, u) .
$$

Our final result concerns first- and second-order necessary conditions for optimality in problem $(P)$, formulated in terms of the adjoint arc $p$ defined by

$$
-\dot{p}(t)^{T}=H_{x}(t)=p(t)^{T} A(t)-L_{x}(t, x(t), u(t)), \quad p(T)=0 .
$$

Theorem 8. Let $(x, u)$ give the minimum in $(P)$, such that the constraint qualification $(C Q)$ holds at $u(t)$ for almost all $t$. Then for all $(y, v)$ satisfying the linearized system

$$
\dot{y}(t)=A(t) y(t)+B(t) v(t), \quad y(0)=0, \quad v(t) \in T_{U(t)}(u(t)),
$$

one has

$$
0 \leq \mathscr{J}_{u}^{\prime}(v)=\int_{0}^{T}\left(L_{x}(t) y(t)+L_{u}(t) v(t)\right) d t
$$


If, in addition, the control set $U(t)$ is convex, then every such pair $(y, v)$ satisfies (19)

$$
\begin{aligned}
0 & \leq \mathcal{F}_{u, 0}^{\prime \prime}(v) \\
& =\int_{0}^{T}\left[L_{2}(t, y(t), v(t))+\max _{\eta \in \Gamma(t)}\left\{v(t)^{T}(\eta G)^{\prime \prime}(t, u(t)) v(t)\right\}+\Psi_{\Sigma(t)}(v(t))\right] d t,
\end{aligned}
$$

where

$$
\begin{aligned}
L_{2}(t, y, v) & =v^{T} L_{u u}(t) v+2 y^{T} L_{u x}(t) v+y^{T} L_{x x}(t) y, \\
\Gamma(t) & =\left\{\eta \in N_{C}(G(t, u(t))): \mathscr{H}_{u}(t)=0\right\}, \\
\Sigma(t) & =\left\{v \in T_{U(t)}(u(t)):\left(p(t)^{T} B(t)-L_{u}(t)\right) v=0\right\} .
\end{aligned}
$$

Proof. The first-order condition $\mathscr{J}_{u}^{\prime}(v) \geq 0$ for all $v$ is a direct consequence of the definition of the epi-derivative. The calculation of $\mathscr{J}_{u}^{\prime}$ implicit in (17) and (18) follows from Theorem 7 and the definition of $E$. Likewise, the secondorder condition $\mathcal{J}_{u, 0}^{\prime \prime}(v) \geq 0$ for all $v$ is obvious: the point of $(19)$ is its formula for the second epi-derivative. This follows from Theorem 7, once we evaluate $w_{1}=-\mathscr{I}_{1}^{\prime}(\boldsymbol{\Phi}(u)) \boldsymbol{\Phi}^{\prime}(u)$. For any $v \in L^{2}$, we write $y=E(v)$ to obtain

$$
\begin{aligned}
\left\langle\mathcal{F}_{1}^{\prime}\right. & \left.(\Phi(u)) \Phi^{\prime}(u), v\right\rangle=\left\langle\mathcal{I}_{1}^{\prime}(\Phi(u)), \Phi^{\prime}(u) v\right\rangle_{L^{2} \times L^{2}} \\
& =\left\langle\mathcal{F}_{1}^{\prime}(x, u),(y, v)\right\rangle_{L^{2} \times L^{2}} \\
& =\int_{0}^{T}\left(L_{x}(t) y(t)+L_{u}(t) v(t)\right) d t \\
& =\int_{0}^{T}\left[\left(\dot{p}(t)^{T}+p(t)^{T} A(t)\right) y(t)+L_{u}(t) v(t)\right] d t \\
& =\int_{0}^{T}\left[\left(\dot{p}(t)^{T} y(t)+p(t)^{T} \dot{y}(t)\right)-p(t)^{T} \dot{B}(t) v(t)+L_{u}(t) v(t)\right] d t \\
& =\int_{0}^{T}\left(L_{u}(t)-p(t)^{T} B(t)\right) v(t) d t \\
& =\left\langle L_{u}-p^{T} B, v\right\rangle .
\end{aligned}
$$

Thus $w_{1}(t)=-\left[\mathscr{F}_{1}^{\prime}(\Phi(u)) \Phi^{\prime}(u)\right](t)=p(t)^{T} B(t)-L_{u}(t)$, and the sets $\Gamma(t)$ and $\Sigma(t)$ of Theorem 7 reduce to those of the current statement.

Remark. If the gradients $g_{i}^{\prime}(t, u(t))$ corresponding to the equality and active inequality indices defining the set $U(t)$ are linearly independent for almost all $t$, then the multipliers $\eta_{i}$ are unique, and Theorem 8 reduces to Loewen and Zheng [7, Theorem 3.4].

\section{REFERENCES}

1. H. Attouch, Variational convergence for functions and operators, Pitman, Boston, 1984.

2. J. P. Aubin and H. Frankowska, Set-valued analysis, Birkháuser, Boston, 1990.

3. F. H. Clarke, Optimization and nonsmooth analysis, Wiley-Interscience, New York, 1983.

4. R. Cominetti, On pseudo-differentiability, Trans. Amer. Math. Soc. 324 (1988), 843-865.

5. C. Do, Generalized second derivatives of convex functions in reflexive Banach spaces, Trans. Amer. Math. Soc. (to appear). 
6. A. Levy, Second-order epi-derivatives of integral functionals with fully amenable integrands, Set-Valued Analysis (to appear).

7. P. D. Loewen and H. Zheng, Generalized conjugate points for optimal control problems, Nonlinear Analysis, Theory Methods \& Applications (to appear).

8. D. Noll, Second order differentiability of integral functionals on Sobolev spaces and $L^{2}$ spaces, Z. Reine Angew. Math. (to appear).

9. R. A. Poliquin and R. T. Rockafellar, $A$ calculus of epi-derivatives with applications to optimization, Canad. J. Math. 45 (1993), 879-896.

10. , Amenable functions in optimization, preprint.

11. R. T. Rockafellar, Integral functionals, normal integrands and measurable selections; in Nonlinear Operators and the Calculus of Variations, Lecture Notes in Math., vol. 543, Springer-Verlag, 1976, pp. 157-207.

12. $\ldots$, First- and second-order epi-differentiability in nonlinear programming, Trans. Amer. Math. Soc. 307 (1988), 75-107.

13. epi-derivatives, Math. Oper. Res. 14 (1989), 462-484.

14. __ Proto-differentiability of set-valued mappings and its applications in optimization, Analyse Non Linéaire (H. Attouch, J.P. Aubin, F. Clarke, I. Ekeland, eds.), 1989, pp. 449-482.

15. $\_$, Generalized second derivatives of convex functions and saddle functions, Trans. Amer. Math. Soc. 320 (1990), 810-822.

16. - Nonsmooth analysis and parametric optimization, Methods of Nonconvex Analysis (A. Cellina, ed.), Lecture Notes in Math., vol. 1446, Springer-Verlag, 1990, pp. 137-151.

Department of Mathematics, University of British Columbia, Vancouver, British Columbia, Canada, V6T 1 Z2

E-mail address: loew@math.ubc.ca

Department of Electrical Engineering, Imperial College of Science, Technology, and Medicine, London SW7 2BT, United Kingdom 